\title{
On the use of LEO satellite constellation for active network management in power distribution networks
}

\author{
Qiang Yang, Member, IEEE, Dave I. Laurenson, Member, IEEE, and Javier A. Barria, Member, IEEE
}

\begin{abstract}
The passive nature of power distribution networks has been changing to an active one in recent years as the number of small-scale Distributed Generators (DGs) connected to them rises. The consensus of recent research is that current slow central network control based upon Supervisory Control and Data Acquisition (SCADA) systems is no longer sufficient and Distributed Network Operators (DNOs) wish to adopt novel management mechanisms coupled with advanced communication infrastructures to meet the emerging control challenges. In this paper, we address this issue from the communication perspective by exploiting the effectiveness of using a Low Earth Orbit (LEO) satellite network as the key component of the underlying communication infrastructure to support a recently suggested active network management solution. The key factors that would affect the communication performance over satellite links are discussed and an analytical LEO network model is presented. The delivery performance of several major data services for supporting the management solution is evaluated against a wide range of satellite link delay and loss conditions under both normal and emergency traffic scenarios through extensive simulation experiments. Our investigation demonstrates encouraging results which suggests that a LEO network can be a viable communication solution for managing the next-generation power energy networks.
\end{abstract}

Index Terms-LEO constellation, satellite, power distribution networks

\section{INTRODUCTION}

$\mathbf{I}$ $\mathrm{N}$ recent years, enormous efforts in the pursuit of lowcarbon energy provision and advances in distributed energy resource (DER) technology have led to a great boom in the utilization of renewable energy sources. Various forms of small-scale DGs, e.g. wind turbines and combined heat and power (CHP) plants, have been connected to power distribution networks. While this presents a revolutionary step in energy generation with many operational benefits, e.g. a great reduction of peak-time demand and minimization of network congestion, the massive penetration of DGs in existing power distribution networks effectively alters its passive nature resulting in bi-directional power flows and several other major adverse impacts, including "voltage rise, increased fault levels, altered transient stability and degradation of protection operation and co-ordination" [1]. This makes the current SCADAbased network control structure with centralized authority at the DNO's control center no longer efficient or practical and calls for novel Active Network Management (ANM) mechanisms.

Alongside existing efforts, e.g. "IntelliGrid" [2] and "SmartGrids" [3], to propose future "intelligent" or "smart" energy networks, AURA-NMS (autonomous regional active network management system) [4] is an ongoing UK effort with its research consortium aiming to exploit a cost-effective ANM solution by implementing a distributed and intelligent control approach through a regional network management system to enhance energy security and quality of supply in the UK medium voltage $(33 \mathrm{kV}$ and $11 \mathrm{kV})$ distribution networks.

The key idea behind AURA-NMS is to devolve the management authority from the centralized utility control center to a set of loosely-coupled regional controllers deployed across the distribution network to carry out management tasks in either an autonomous or a cooperative fashion with controllers governing individual pre-defined control regions. The deployed regional controllers are expected to manage their local power network devices as well as support collaborative decisionmaking with peer controllers across multiple regions. The underlying communication infrastructure plays a central role in the realization of such a scheme.

Our earlier investigations [5]-[7] presented the conceptual communication architecture in AURA-NMS and identified the requirements and engineering challenges. They concluded that current SCADA systems can barely meet the requirements of the basic control algorithms due to limitations in many aspects, e.g. constrained data-rate (a few hundred to a few thousand bits per second), inflexible device organization (e.g. multi-drop or daisy-chained lines), lack of communication infrastructure in the majority of low voltage sites, poor scalability of technologies and limited interoperability due to standard and protocol inconsistency. We highlighted the necessity of adopting a unified information model based on emerging industrial standards (e.g. IEC 61970, IEC 61968 and IEC 61850) to enable seamless and transparent communication between the various voltage levels across the overall power distribution grid. In addition, we pointed out the trend that the future "power utility Intranet" will be, almost certainly, independent of the public Internet. However, it will be IP-based due to technology maturity, enhanced interoperability, low-cost and easy migration path, as suggested by many researchers (e.g. [8], [9]). These findings motivate our further exploration of new communication infrastructures with reliable, flexible and future-proof technologies to enable autonomous as well as collaborative network control proposed by AURA-NMS.

Satellite networks have become one of the key components of today's communication infrastructure and have been proved capable of reliably delivering a variety of services for businesses (e.g. satellite TV) and industries (e.g. remote telemetry and global positioning). In addition, due to its several unique characteristics, it is expected to be a key part of the communication infrastructure for supporting the management of future energy networks:

1) The power distribution networks are generally geograph- 
ically large, including rural, urban, suburban and some very remote sites covering customers numbering a few million to a few thousand or even fewer with different degrees of communication availability. Satellites can reach and provide data service over all these areas regardless the geographical conditions.

2) Current communication infrastructures in utilities were built with the most available technologies at the time resulting in an end-to-end path consisting of channels with diverse communication media and physical characteristics (e.g. leased digital fibre, private wire, telephone lines, satellite and mobile radio). Frequent transitions between different media degrade the communication availability and reliability. Adopting a satellite based system as the key technology effectively removes such deleterious impacts.

3) The defined control regions across the power distribution network may be very diverse in terms of the number and type of network elements, e.g. DGs, substation transformers and feeders, which results in data traffic over the underlying communication system with different demands and characteristics. Satellite networks have high flexibility and efficiency in bandwidth utilization to meet the requirements of bandwidth-on-demand capability and dynamic channel allocation.

4) Current power distribution networks continue to grow with an increasing number of monitoring and control devices. Satellite networks are flexible enough for easy expansion and reconfiguration to incorporate and manage communication with newly added power network devices by simply installing system interfaces at device premises with a low installation and maintenance cost.

\section{RELATED WORK}

Satellite-based systems have been adopted in power utilities for years and the majority of the research effort (e.g. [10][12]) has focused on the use of Geo-stationary (GEO) satellites as a part of the communication infrastructure to provide communication services in power utilities.

In [10], a terrestrial-GEO satellite hybrid communication infrastructure is proposed to support dedicated data services for power utilities. In [11], the authors discussed the use of a satellite-based system for wide-area measurement, command and control of power systems and suggested its application to improve the dynamic thermal rating of overhead transmission circuits. In [12], the authors reviewed the incorporation of GEO satellites into existing monitoring, protection and control systems in Energy Management Systems (EMS) and SCADA systems of power utilities. In the aforementioned proposals, the satellite component is expected to operate in areas where terrestrial communication is not available. As a result, it plays a complementary rather than a central role in the overall infrastructure as many critical aspects of GEO technology, notably excessive propagation delay (approximately $250 \mathrm{~ms}$ ) and high costs, have an effect on the overall performance. On the other hand, recent advances in LEO satellite systems provide several outstanding advantages over GEO based systems, such as greatly reduced propagation distance, small antenna size, lower power consumption, smaller signal attenuation, lower cost, minimal impact due to satellite failure and better support of IP data services.

Although LEO networks have been studied from many aspects, including resource management (e.g. [13]), routing mechanisms (e.g. [14]), transport protocol performance evaluation (e.g. [15]), and service level agreement (SLA) guarantees (e.g. [16]), few studies have addressed the application of LEO networks as the key component of the communication infrastructure for managing large critical systems. The application of LEO satellites for remote meter reading and rural distribution automation based on its two-way communication capability is proposed in [17]. However, no performance assessment results and analysis are presented. In [18] the authors assessed the IP data service of a specific LEO constellation - "Globalstar" (orbit altitude: $1,410 \mathrm{~km}$, data rate: $7.2 \mathrm{~kb} / \mathrm{s}$ ) which was designed to support low rate voice and data services through an experimental test-bed with a prototype Intelligent Electronic Device (IED). It concluded that while the Globalstar based LEO network is viable for supporting general network automation, it barely meet the stringent delay requirements for time critical applications in smart distribution networks due to its limited bandwidth and high orbit altitude. It should be highlighted that the conclusion drawn in previous work is merely based on studies of specific LEO constellations which cannot provide the knowledge of LEO network performance for a range of channel delay and loss scenarios under different power network operational conditions (normal and emergency). In this paper, without limiting the study to specific LEO constellations, we develop a generic methodology to assess the effectiveness of a LEO network for providing IP based data services for an AURA-NMS type of approach (shown in Figure 1(b)). The main contributions of this paper are summarized as follows:

1) A generic analytical model for a LEO network is presented with an analysis of network delay and loss behavior.

2) A set of IP data services with diverse traffic characteristics and patterns over LEO networks are evaluated for both regular and emergency operational scenarios under a range of delay and loss conditions.

The obtained full performance profile from this study can be used to justify the effectiveness of LEO networks for AURANMS operation. The remainder of the paper is organized as follows: Section III briefly introduces the AURA-NMS solution. Section IV overviews the LEO satellite network, discusses its relevant characteristics that may affect its performance and presents the analytical model of the LEO network and the delay analysis for the network. Section V sets up the network and traffic scenarios for simulation experiments and provides numerical results and analysis. Finally, some conclusions and remarks are made in Section VI.

\section{AURA-NMS APPROACH}

Figure 1 illustrates the current centralized control (Figure 1(a)) and suggested AURA-NMS based approach (Figure 1(b)) 


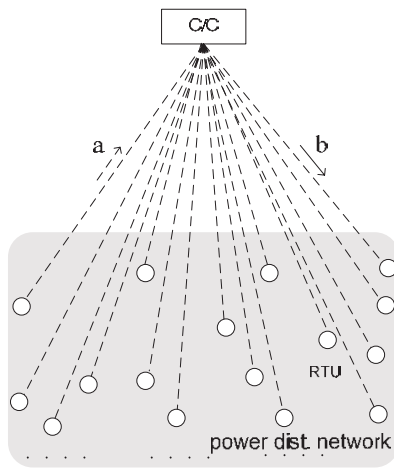

(a)

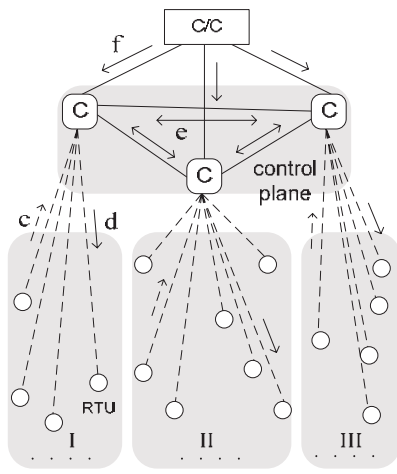

(b)
Fig. 1: Two control solutions in $11 \mathrm{kV}$ network of a UK DNO: (a) centralized control; (b) AURA-NMS approach

by using the $11 \mathrm{kV}$ distribution network of a UK DNO as an example. The centralized control relies on the DNO's SCADA system connecting the field devices and the DNO's control center ("C/C") for network monitoring and control tasks (Figure 1: flows a and b). In the AURA-NMS approach, hardware controllers ("C") are installed in individual predefined control regions (I, II and III) which can access their local sensing and actuating devices via the communication connections between them and the network monitoring and control actions are taken locally (Figure 1: flows $\mathrm{c}$ and d). These networked regional controllers form the computation and communication platform for the execution of a set of active network management functions to conduct a range of network management tasks, for example: voltage control [19] to maintain voltage within the regulated limits under conditions of load variation or intermittent output of DGs; power flow management [20] to operate the network within thermal limits at a given level of DG generation and contractual constraint; and automatic fault management to restore power supply to as many customers as quickly as possible after a fault occurs.

The credibility of derived control solutions from these algorithms firmly relies on the regional controllers being aware of the network operational states, including feeder voltage, current, DG outputs, transformer information, loads, and so forth. In power distribution networks, substation Remote Telemetry Units (RTUs) directly interface with the physical power network and collect these measurements from field sensors (analogue and digital) and forward control signals to actuating devices. The snapshots of network operation over time can be obtained through polling RTUs in a non-continuous fashion with a reasonable polling rate. In addition, urgent field events or notifications detected by the RTU, e.g. alarms, are expected be delivered immediately to the controllers as an unsolicited data service in a random manner. At the controller, collected network state information is analysed to detect any operating conditions (e.g. voltage excursion) that may require a corrective action (e.g. operating an on load tap changer). One, or a combination of multiple different control signals, could be issued to control the actuating devices via the RTU. For example, the taps may be moved up in a transformer whilst reducing real and reactive power of a DG.

The AURA-NMS approach is both autonomous and collaborative: when the scope of the detected anomalous condition, and its solution, does not exceed the boundary of the region, the controller will carry out local control autonomously without consulting other controllers. However, autonomous control may be insufficient and wide-area control may be needed in the case that adjacent regions could be affected or potential control conflicts are detected. In such cases the controllers in these adjacent regions only have a partial view of the problem, therefore they will need to cooperate to derive suitable solutions through coordination (Figure 1: flow e). AURA-NMS suggests combining multi-agent theory into the management framework by encapsulating and distributing the management functions as agents over different hardware controllers to accomplish complex tasks [21]. Coordination is realized through agent message exchange between peer controllers. It is worth noting that although the controllers are expected to control the distribution network, they are still governed by the DNO's control center (operator) to ensure that the overall operation meets certain utility objectives (Figure 1: flow f).

\section{LEO Network CHARACTERISTICS AND Modeling}

In general, a LEO network consists of a number of satellites organized as a constellation in orbits of 500-2,000 km above the earth's surface. Communication between any two points within the constellation coverage is possible via the LEO network at any time. There are two classes of LEO networks, namely "little LEO" and "big LEO", which are launched to support a wide range of communication services. The former, such as Orbcomm, Starnet, and Leosat, offer shortrange narrowband non-voice communication, e.g. paging, vehicle tracking, messaging and environmental monitoring. The latter, including Iridium, Globalstar and Odyssey, focus on delivering real-time voice to personal handsets and low bit rate data services. A more detailed description of these LEO service providers can be found in [22]. Some proposals, e.g. the Teledesic system (orbit altitude: $700 \mathrm{~km}$, data rate: tens of $\mathrm{Mb} / \mathrm{s}$ ), aim to provide a global "Internet-in-the-Sky" to offer Internet access for high quality broadband service. We consider that the available or future LEO networks with ubiquitous coverage, generous bandwidth, low orbit altitude and affordable service tariff are particularly attractive for management of future energy networks, e.g. the Teledesic-like LEO constellations.

\section{A. LEO Network Communication Characteristics}

With respect to the control of power utilities, the key communication characteristics that would affect network management performance are latency, bandwidth, packet loss due to congestion, and packet corruption due to transmission errors. In a LEO constellation these parameters can exhibit dynamics over time due to intrinsic features of LEO networks which are discussed as follows.

Latency: the three main components are the propagation and switching delay, the transmission delay and the queuing delay. 
In a LEO constellation, the dominant part is the propagation delay which depends upon the distance the signal traverses between the source and the destination satellites. In addition, due to the relative satellite motion, the handover process (transferring a connection between satellites which happens approximately every 8 to 11 minutes [23]) and routing path changes, cause the overall delay characteristic between two ground stations in a LEO constellation to vary over time. In [24] TCP performance was investigated showing that roundtrip time varied in a range of patterns including sudden changes due to path changes. It concluded that no drastic impacts are observed as the use of a retransmission timeout (RTO) timer with a large minimum value can effectively avoid packet retransmission events. Therefore, we neglect the delay variation and assume that the propagation delay is constant during the overall communication process.

Channel access and bandwidth asymmetry: many advanced Medium Access Control (MAC) protocols are available taking advantage of both random access and reservation protocols with greatly improved scalability and flexibility. These exhibit excellent throughput and channel access characteristics [25]. In addition, many designed LEO networks demonstrate asymmetric bandwidth on the forward and backward paths with distinct data rates due to economic factors, e.g. satellite terminals are able to receive data at tens of $\mathrm{Mb} / \mathrm{s}$ but the data sending rate is limited to several hundred $\mathrm{kb} / \mathrm{s}$ or even lower.

Transmission errors: in wired networks, almost all the packet loss is due to buffer overflow caused by congestion. However, an occasional high bit error rate (BER) over satellite channels can cause packet corruption and ultimately discard if it cannot be recovered at the end point. TCP deems this as an indication of communication congestion, and in turn undergoes a number of unnecessary switches to the slow-start phase with degraded throughput. In general, a satellite link exhibits a BER of as low as $10^{-8}$ on average and $10^{-4}$ in the worst case. Advanced modulation and coding techniques are often adopted to obtain even lower BER to deliver "fibre-like" error rates most of the time.

Communication congestion: inter-satellite links (ISLs) in most LEO networks use high-frequency radio or optical links which have a high bandwidth, e.g. $25 \mathrm{Mb} / \mathrm{s}$ in Iridium and 155 $\mathrm{Mb} / \mathrm{s}$ in Teledesic. The bandwidth bottleneck resides at the links between the ground stations and their serving satellites, known as User Data Links (UDLs), due to restricted spectrum. Therefore, heavy congestion is generally not present on ISLs.

In summary, we envisage that future LEO satellite networks can be characterized by low BERs, high channel bandwidth with asymmetry, low but varied propagation delays (compared with GEO based systems), minimal channel access delay and low congestion within the constellation. Our following analysis and performance assessment are based on these assumptions.

\section{B. LEO Network Model}

Consider a polar-orbiting LEO satellite constellation consisting of $N$ evenly separated (angular separation of $180^{\circ} / N$ ) polar orbits (planes $p_{1}, p_{2}, \ldots, p_{N}$ ) which cross each other only over the pole areas and each orbit has $M$ evenly separated satellites (angular separation of $360^{\circ} / M$ ). In this paper, we assume that each satellite, $s_{i, j}$ (the $j^{\text {th }}$ satellite on the $i^{t h}$ plane), in the constellation has four bidirectional inter-satellite links (ISLs) with its four immediately neighboring satellites: two intra-plane ISLs connecting to vertically adjacent satellites in the same plane, $s_{i, j \pm 1}$, and two inter-plane ISLs connecting to the closest neighboring satellites in the adjacent planes, $s_{i \pm 1, j}$. As shown in Figure 2, these ISLs forms a mesh network with a "seam" between the first plane $\left(p_{1}\right)$ and last plane $\left(p_{N}\right)$, where satellites in planes along the seams rotate in opposite directions.

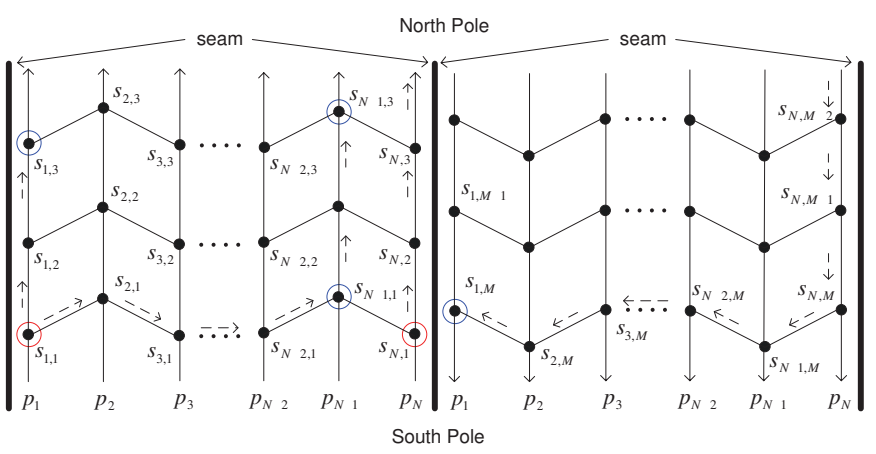

Fig. 2: A polar LEO satellite constellation with 4 ISL connections

On the same plane, all satellites move in the same circular direction. The intra-plane ISLs are maintained at all times and the length (and hence the propagation delay) are fixed at all times [14].

$$
L_{I S L}=\sqrt{2} \cdot(R+h) \cdot \sqrt{1-\cos \left(\frac{360^{\circ}}{M}\right)}
$$

Where $R$ and $h$ are the earth radius $(6378 \mathrm{~km})$ and LEO orbital altitude, respectively.

Between planes, inter-plane ISLs cannot always be maintained and are generally considered as unavailable under two circumstances: (1) when an inter-plane ISL is between counterrotating satellites across the "seam" even though they may be very "geographically" close; (2) when two satellites in adjacent planes move into the Polar Regions. The length of inter-plane ISLs (and hence the propagation delay) varies over time with the satellite movement and is related to the latitude $(X)$ at which the inter-plane ISL resides [14]

$$
\bar{L}_{I S L}=\sqrt{2} \cdot(R+h) \cdot \sqrt{1-\cos \left(\frac{360^{\circ}}{2 \cdot N}\right)} \cdot \cos X
$$

It can be seen that $\bar{L}_{I S L}$ is longest when satellites are over the equator $\left(X=0^{\circ}\right)$ and shortest when they are near the Polar Regions $\left(X \approx 90^{\circ}\right)$.

The topological pattern in a LEO satellite constellation changes over time with the satellite movement. Therefore, efficient routing mechanisms are generally required to select suitable end-to-end paths for connections and also maintain the connectivity throughout the communication process. If the source and destination ground stations are not within the same satellite footprint, it causes packets to take multiple hops 
from the source satellite to the destination satellite within the constellation. Given the source-destination satellite pair $\left(s_{i s, j s}, s_{i d, j d}\right)$, the propagation distance in terms of number of hops, $L_{p}$, can be expressed as follows:

$$
L_{p}=\min \left\{\left|j_{d}-j_{s}\right|, M-\left|j_{d}-j_{s}\right|\right\}+\left|i_{d}-i_{s}\right|
$$

Compared with GEO systems, the lower altitude of a LEO satellite constellation implies a significantly smaller footprint per satellite which means more satellites are needed for global coverage. According to [26], the approximate number of satellites, $N \cdot M$, to obtain continuous global coverage between any latitude $X$ and the pole can be obtained by equation 4 . The number of satellites needed as a function of orbital altitude $h(600 \mathrm{~km}$ to $2000 \mathrm{~km})$ is presented in Table I (assuming $\left.\theta=10^{\circ}\right)$.

$$
\begin{aligned}
& N \cdot M \cong \frac{4 \cdot \cos X}{1-\cos \varphi} \\
& 1.3 \cdot N<M \cdot \cos X<2.2 \cdot N \\
& \varphi=\arccos \left(\frac{R}{R+h} \cdot \cos \theta\right)-\theta
\end{aligned}
$$

Where $\varphi$ and $\theta$ are the Earth-centred half-cone-angle of coverage for each satellite and the minimum elevation angle, respectively.

TABLE I: The number of LEO satellites for global coverage

\begin{tabular}{|c|ccc|}
\hline$h(\mathrm{~km})$ & $N$ & $M$ & $N \cdot M$ \\
\hline 600 & 7 & 15 & 105 \\
800 & 7 & 11 & 77 \\
1000 & 6 & 10 & 60 \\
1200 & 6 & 8 & 48 \\
1400 & 5 & 8 & 40 \\
1600 & 5 & 7 & 35 \\
1800 & 4 & 8 & 32 \\
2000 & 4 & 7 & 28 \\
\hline
\end{tabular}

Based on the previous analysis, we further present an analytic communication model characterizing the packet delay and loss behavior on the end-to-end path (including both terrestrial and space segments) between two communication terminals. This model does not incorporate the delay variation due to topology and routing changes or other LEO dynamic characteristics. For sake of clarity, the following notation is used:

Figure 3 illustrates the communication infrastructure, including LEO constellation, UDLs, gateways (acting as access points) and the terrestrial segment. The packet delay on the end-to-end path is comprised of the delay that occurs in both terrestrial and LEO segments:

$$
D=D_{T}+D_{S}
$$

The delay in the terrestrial segment and LEO network can be expressed as follows:

$$
\begin{gathered}
D_{T}=d_{T, t}+d_{T, p}+d_{T, s}+d_{T, q} \\
D_{S}=d_{S, t}+d_{S, p}+d_{S, s}+d_{S, q}
\end{gathered}
$$

$\begin{array}{ll}D & \text { Packet delay on end to end path } \\ D_{T} & \text { Terrestrial network packet delay } \\ d_{T, t} & \text { Terrestrial network packet transmission delay } \\ d_{T, p} & \text { Terrestrial network packet propagation delay } \\ d_{T, s} & \text { Terrestrial network packet switching and processing delay } \\ d_{T, q} & \text { Terrestrial network packet queuing delay } \\ D_{S} & \text { LEO network packet delay } \\ d_{S, t} & \text { LEO network packet transmission delay } \\ d_{S, p} & \text { LEO network packet propagation delay } \\ d_{S, s} & \text { LEO network packet switching and processing delay } \\ d_{S, u p} & \text { LEO network uplink delay } \\ d_{S, d o w n} & \text { LEO network downlink delay } \\ d_{S, I S L} & \text { LEO network inter-satellite link delay } \\ d_{S, q} & \text { LEO network packet queuing delay } \\ L_{L E O} & \text { Packet propagation distance within LEO constellation } \\ P_{S} & \text { Packet size (in bits) } \\ S_{c a p} & \text { LEO satellite channel capacity } \\ S_{v e l} & \text { Signal propagation velocity (nearly the speed of light) } \\ S_{B E R} & \text { Satellite channel BER } \\ P_{L, e} & \text { Packet loss rate due to transmission errors } \\ P_{L, c} & \text { Packet loss due to network congestion } \\ h & \text { LEO orbital altitude }\end{array}$

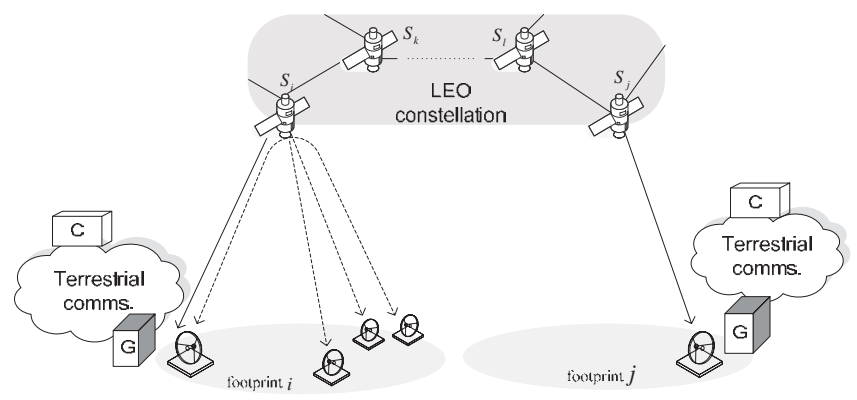

Fig. 3: End-to-end communication infrastructure including a LEO satellite constellation

This work assumes that high-speed and reliable terrestrial links (e.g. ADSL or optical fibres) are adopted to connect with the field equipment in power distribution networks. Therefore, significant queuing delay $\left(d_{T, q}\right)$ may occur on UDLs. Other delay components $\left(d_{T, t}, d_{T, p}\right.$ and $\left.d_{T, s}\right)$ in the terrestrial segment can be one or several magnitudes less, and therefore are negligible.

In the satellite segment, the transmission delay $\left(d_{S, t}\right)$ is determined by the satellite channel capacity, $S_{c a p}$ and the packet size, $p_{s}$. The propagation delay $\left(d_{S, p}\right)$ further consists of delays on up $\left(d_{S, \text { up }}\right)$ and down $\left(d_{S, \text { down }}\right)$ UDLs and the inter-satellite link delay $\left(d_{S, I S L}\right)$. In addition, as packets may pass through several satellites within the constellation, they will suffer additional queuing $\left(d_{S, q}\right)$, switching and processing $\left(d_{S, s}\right)$ delays at each hop. Therefore, $D_{S}$ can be further expressed as

$$
D_{S}=\frac{p_{s}}{S_{c a p}}+\frac{2 \cdot h}{S_{v e l}}+\frac{L_{L E O}}{S_{v e l}}+d_{S, s}+d_{S, q}
$$

In LEO networks, packet transmission delay is very small and the buffer delay within the constellation can be disregarded due to minimal inter-satellite congestion. Thus, we only consider two dominating components that contribute to the 
end-to-end delay: switching time and signal propagation time. The former is proportional to the number of satellites that the packet traverses on the path which depends on the satellite constellation and adopted routing method. The latter depends on the orbital altitude and the number of ISL links.

As shown in Table I, it is clear that the number of satellites required to provide global coverage decreases with increasing satellite altitude. Hence, a higher altitude reduces the switching delay component but leads to increased propagation delay. In [27], the authors discussed the impact of LEO orbital altitude selection on communication performance. In our study, we are particularly interested to find out the upper and lower delay bounds of LEO networks and further assess the data communication services between these delay bounds for supporting AURA-NMS operation.

The best scenario occurs when source and destination terminals are within the same satellite footprint (i.e. no ISL links), and therefore it comprises only the up and downlink propagation time and a single satellite switching delay. The worst case happens when the terminals are on adjacent planes but separated by one of the two "seams", across which there are no inter-satellite links, the packet has to traverse over one of the poles to reach the destination. The maximum propagation length (no. of hops, $L_{\max }$ ) and involved number of satellites, $S_{\max }$, within LEO constellation are given in equations (9) and (10).

$$
\begin{gathered}
L_{\max }=(N-1) \cdot \bar{L}_{I S L}+\left\lfloor\frac{M}{2}\right\rfloor \cdot L_{I S L} \\
S_{\max }=N+\left\lfloor\frac{M}{2}\right\rfloor
\end{gathered}
$$

Based on the presented analytical model, Table II shows the calculated minimum and maximum propagation and switching delay in LEO networks with different altitudes (600-2000 $\mathrm{km}$ ). Therefore, our following simulation experiments (see section $\mathrm{V}$ ) for assessing data delivery performance based on the configuration that the propagation and switching delay in LEO network is within the range of $10 \mathrm{~ms}$ to $200 \mathrm{~ms}$.

Following the analysis of the end-to-end packet delay, another key aspect affecting the performance is packet loss. Two major types of loss are considered, namely packet congestion loss due to buffer overflow in communication elements $\left(P_{L, c}\right)$ and corruption loss $\left(P_{L, e}\right)$ due to channel transmission errors. In general, satellite channels suffer both random and burst errors. The burst errors are mainly due to convolution encoding schemes and are not considered in this paper. We assume that bit errors are independent and identically distributed (iid) as in the literature (e.g. [28]) and that the packet error rate can be related to the channel BER $\left(S_{B E R}\right)$ and the packet size $\left(p_{s}\right)$ by the following formula:

$$
P_{L, e}=1-\left(1-S_{B E R}\right)^{p_{s}}
$$

\section{Performance Evaluation And Numerical RESULTS}

\section{A. Data Traffic Modeling}

To design a suitable communication provision for AURANMS, the different types of data traffic along with their characteristics need to be understood. We consider four major traffic types:

1) Network monitoring: network measurements (packets of 32 bytes) collected by RTUs are periodically acquired by designated regional controllers with a pre-defined polling rate.

2) Network alarms: urgent field events or notifications (packets of 60 bytes) should be reliably delivered to controllers immediately once generated.

3) Control commands: control signals (packets of 60 bytes) issued by regional controllers are delivered to the actuating devices via the RTU to take certain actions, where a single control solution may consist of more than one control packets. It is assumed that the number of control packets is uniformly distributed between 1 and 10 .

4) Coordination traffic: the controllers coordinating with peers in finding control solutions exchange a certain number of ACL messages (packets of 1000 bytes).

These traffic types are expected to be delivered in different time scales to fulfil their functions. The general requirements of data delivery are briefly described in [28] as "slow for post-event analysis, near real-time for monitoring and as close to real-time as possible for control or protection". This implies that in current "best-effort" communication infrastructure without inherent service prioritization or bandwidth reservation, selecting suitable transport protocols for different traffic types becomes paramount. It is concluded in [9] that the performance of using TCP to deliver critical real-time information is unsatisfactory due to its congestion adaptation behavior and a modified UDP with reliability enhancement is proved to be suitable. This is also confirmed by [8] as it introduced a UDP based protocol ("Astrolabe") for network monitoring.

In this paper, we aim to investigate the standard IP based transport protocols for supporting these data services over a LEO network. For network monitoring traffic, we emphasize its timeliness and consider that an absolute data delivery guarantee is not essential when the sampling rate is high, e.g. one every several seconds. In respect to alarms and control commands, they require reliable delivery and can tolerate reasonable delay, e.g. on-load tap changers act on the time-scale of tens of seconds, and thermal conditions (e.g. transformer overheating) may to be tolerated for several minutes before a control action is taken. Therefore, network monitoring data is carried by UDP, while alarms and control commands are carried by TCP with delivery acknowledgements and retransmission upon packet loss (each event initiating a connection setup). In addition, the software agents operating across regional controllers use HTTP/TCP as the underlying message transport protocol [29] for cooperative decision-making.

The primary performance metric is defined as data delivery duration which is interpreted as the total time to complete a measurement collection cycle, a control command delivery or a coordination process. The delivery time of data acquisition and control (and alarm) outside the substation is suggested to be within $1 \mathrm{~s}$ [36]. In this work, to evaluate the feasibility and potential of using LEO based communication infrastructure 
TABLE II: Minimum and maximum LEO propagation and switching delay, ms (assuming switching delay is 3 ms per satellite)

\begin{tabular}{|c|ccc|ccc|}
\hline$h$ & $d_{S, p^{*}}$ & $d_{S, s^{*}}$ & $d_{S, p}+d_{S, s^{*}}$ & $d_{S, p}{ }^{* *}$ & $d_{S, s}{ }^{*}$ & $d_{S, p}+d_{S, s}{ }^{* *}$ \\
\hline 600 & 4.0 & 3.0 & 7.0 & 133.8 & 42.0 & 175.8 \\
800 & 5.3 & 3.0 & 8.3 & 136.6 & 36.0 & 172.6 \\
1000 & 6.7 & 3.0 & 9.7 & 146.3 & 33.0 & 179.3 \\
1200 & 8.0 & 3.0 & 11.0 & 150.7 & 30.0 & 180.7 \\
1400 & 9.3 & 3.0 & 12.3 & 152.8 & 27.0 & 179.8 \\
1600 & 10.7 & 3.0 & 13.7 & 145.6 & 24.0 & 169.6 \\
1800 & 12.0 & 3.0 & 15.0 & 158.1 & 24.0 & 182.1 \\
2000 & 13.3 & 3.0 & 16.3 & 150.2 & 21.0 & 171.2 \\
\hline
\end{tabular}

Note: Best scenario $(*)$; worst scenario $(* *)$

for supporting the AURA-NMS based control solution in medium voltage $(33 \mathrm{kV}$ and $11 \mathrm{kV})$ distribution networks, we directly adopt this requirement in the assessment of $33 \mathrm{kV}$ network. At the lower voltage level, the events in $11 \mathrm{kV}$ network are generally considered less critical, and hence the requirement is set to $10 \mathrm{~s}$. For the same reason, the time requirement for coordination between peer-controllers in the 33 and $11 \mathrm{kV}$ network are set to $10 \mathrm{~s}$ and $100 \mathrm{~s}$, respectively, as adopted in our previous investigation [5]. Extensive simulation experiments based on $n s 2$ [31] are carried out to assess performance against a range of LEO propagation and switching delay $(10 \sim 200 \mathrm{~ms})$ and channel packet error rate $\left(10^{-8} \sim 10^{-4}\right)$ conditions for network monitoring, alarms, control and coordination traffic under normal and emergency operational conditions. The results are presented as contour graphs with numbers on contour lines representing the 95th percentile of the data delivery duration values (in seconds) corresponding to a given LEO delay and packet error rate. All experiments are conducted with a simulation time of 10000 seconds (about 2.8 hours) to capture steady state performance measurements.

\section{B. Numerical Results}

We consider the bandwidth asymmetry of LEO channels for carrying monitoring and control traffic with a forward channel (controller to RTU) capacity of $2048 \mathrm{~kb} / \mathrm{s}$ (E1 link) and backward channel (RTU to controller) capacity of $16 \mathrm{~kb} / \mathrm{s}$ or $64 \mathrm{~kb} / \mathrm{s}$. The buffer size of intermediate communication nodes (ground station and satellites) is set to 50 packets for all experiments. Under the normal operational condition, the periodicity and volume of RTU data delivery to the controller are assumed to be every $10 \mathrm{~s}$ and 1000 bytes respectively. The field alarms and control events are generated randomly following a uniform distribution with arrival intensity, $\lambda$, of 0.02 and 0.005 , which are equivalent to the occurrence of alarm and control events every $50 \mathrm{~s}$ and $200 \mathrm{~s}$ on average. For network monitoring, alarms and control data packets, the LEO channel BER of $\left(10^{-8}, 10^{-4}\right)$ can be translated into a packet error rate of $(0,0.06)$ based on equation (10).

Figure 4 shows the data delivery duration result for data retrieval from a single RTU against the LEO propagation and switching delay (from $10 \mathrm{~ms}$ up to $200 \mathrm{~ms}$ ) and the packet error rate of $(0,0.06)$ for two different backward channel capacities, $16 \mathrm{~kb} / \mathrm{s}$ (filled contour plot) and $64 \mathrm{~kb} / \mathrm{s}$ (solid lines). The number shown on each contour line represents the 95th percentile (applied to all the following figures) of the data delivery duration of all the data acquisition events, e.g. for the $64 \mathrm{~kb} / \mathrm{s}$ channel, statistically $95 \%$ of the RTU data acquisition can be completed within about $0.3 \mathrm{~s}$ when the LEO delay is $50 \mathrm{~ms}$. The results demonstrate that the network monitoring duration increases along with the delay increases in LEO network, and the impact of channel errors is minimal. This is because UDP has no retransmission mechanism to cope with packet loss due to transmission errors. It also shows that a $64 \mathrm{~kb} / \mathrm{s}$ channel has a much more desirable performance, e.g. 1000 bytes of data can be retrieved within less than $0.45 \mathrm{~s}$ in the worst case scenario, which easily meets the delivery time requirements for both $33 \mathrm{kV}$ and $11 \mathrm{kV}$ networks.

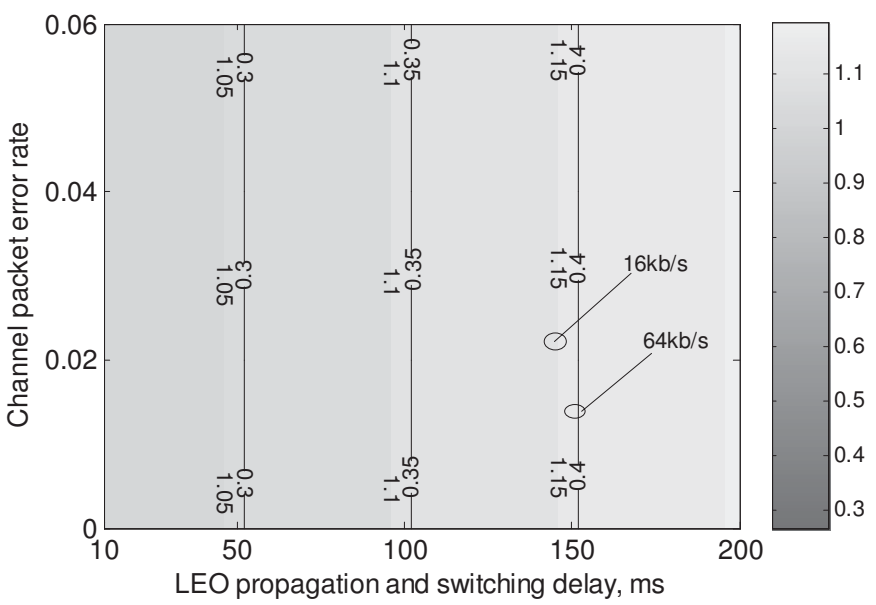

Fig. 4: Data acquisition duration: $16 \mathrm{~kb} / \mathrm{s}$ (filled contour plot), $64 \mathrm{~kb} / \mathrm{s}$ (solid lines)

Figure 5 illustrates the data delivery duration for sending a control solution to the RTU consisting of one or more control packets. Keeping the same previous channel asymmetry configuration, the result demonstrates the LEO network performance degrades when delay and packet error rate increase. Any erroneous packet is treated as an indication of link congestion and consequently causes TCP to decrease the data rate to avoid further congestion and retransmissions following the error detection need to take several roundtrips. The result shows that backward channels with two different capacities $(16 \mathrm{~kb} / \mathrm{s}$ and $64 \mathrm{~kb} / \mathrm{s})$ perform similarly if the same E1 forward channel is used. The latter performs slightly better when the delay is small as its higher bandwidth results in a faster acknowledgement of control packet receipt. However, with large delay (greater than $150 \mathrm{~ms}$ ) and error rate (greater than 0.02 ), the $64 \mathrm{~kb} / \mathrm{s}$ backward channel performs worse primarily due to the combined effect of large bandwidth-delay, 
excessive retransmission upon data corruption and other data traffic (i.e. alarms, RTU data acquisition). It can be seen that the data delivery duration of a control solution (consisting of up to 10 control packets) can be within $1 \mathrm{~s}$ when the delay and error rate are small (e.g. $50 \mathrm{~ms}$ and 0.02) and no more than $10 \mathrm{~s}$ in the worst scenario (i.e. delay of $200 \mathrm{~ms}$ and error rate of 0.06). Such performance meets the delivery time requirement for the $11 \mathrm{kV}$ distribution network imposed by an AURA-NMS type of approach. It also indicates that advanced physical layer, MAC or error control mechanisms for handling errors are needed to enhance the communication performance once deployed in the $33 \mathrm{kV}$ network.

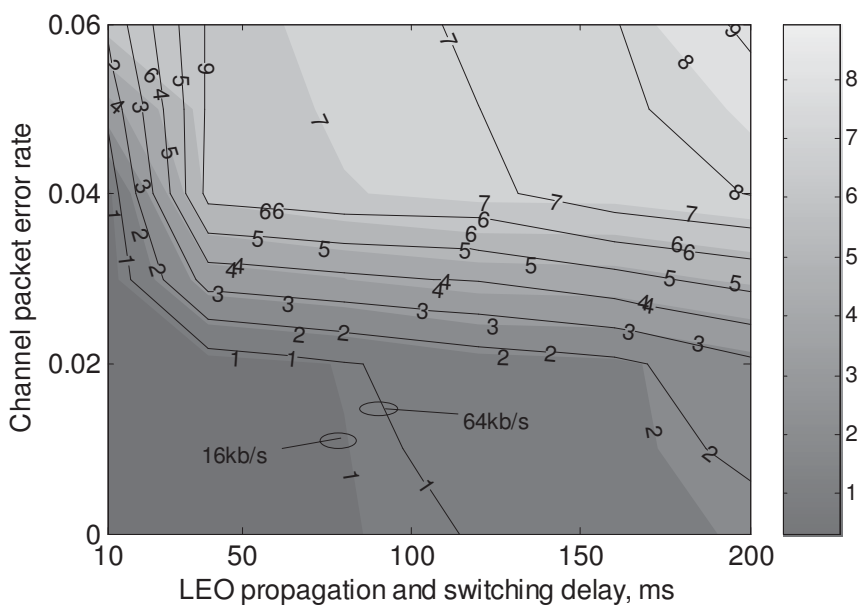

Fig. 5: Control command delivery duration $\left(\lambda=0.005 \mathrm{~s}^{-1}\right)$ : $16 \mathrm{~kb} / \mathrm{s}$ (filled contour plot), $64 \mathrm{~kb} / \mathrm{s}$ (solid lines)

Figure 6 illustrates the duration result of the field alarm delivery for the satellite backward channel capacity of $16 \mathrm{~kb} / \mathrm{s}$ and $64 \mathrm{~kb} / \mathrm{s}$, respectively. Each alarm message is assumed to be a single packet. It shows that the $64 \mathrm{~kb} / \mathrm{s}$ channel outperforms the $16 \mathrm{~kb} / \mathrm{s}$ channel at all times, particularly when the delay is small (less than $75 \mathrm{~ms}$ ) as the negative impact of delay on bandwidth utilization efficiency is minimized. It also shows that the $64 \mathrm{~kb} / \mathrm{s}$ channel can deliver the alarm within $1 \mathrm{~s}$ when the channel packet error rate is sufficiently low (e.g. less than 0.001 ) and always within $10 \mathrm{~s}$ even in the worst case scenario.

Now we look into the coordination of peer controllers which involves agent message exchanges. The satellite channel BER $\left(10^{-8}, 10^{-4}\right)$ results in a packet error rate in the range of $(0,0.10)$ for agent messages. Here we consider the channel is symmetric and two channel capacities, $1024 \mathrm{~kb} / \mathrm{s}$ and $2048 \mathrm{~kb} / \mathrm{s}$ (E1), are examined for exchanging 200 messages between any pair of peer regional controllers with the coordination event following a uniform distribution with arrival intensity of 0.005 (i.e. on average 1 event every 200 s). Figure 7 shows the data delivery duration to complete a process of message exchange against packet error rate from 0 up to 0.1 and delay from $10 \mathrm{~ms}$ to $200 \mathrm{~ms}$. Again, the performance degrades when the delay and packet error rate increase. Significant performance improvement can be observed by using the E1 channel when the LEO network delay and channel error rate are low. However, the benefit of using an E1 link becomes

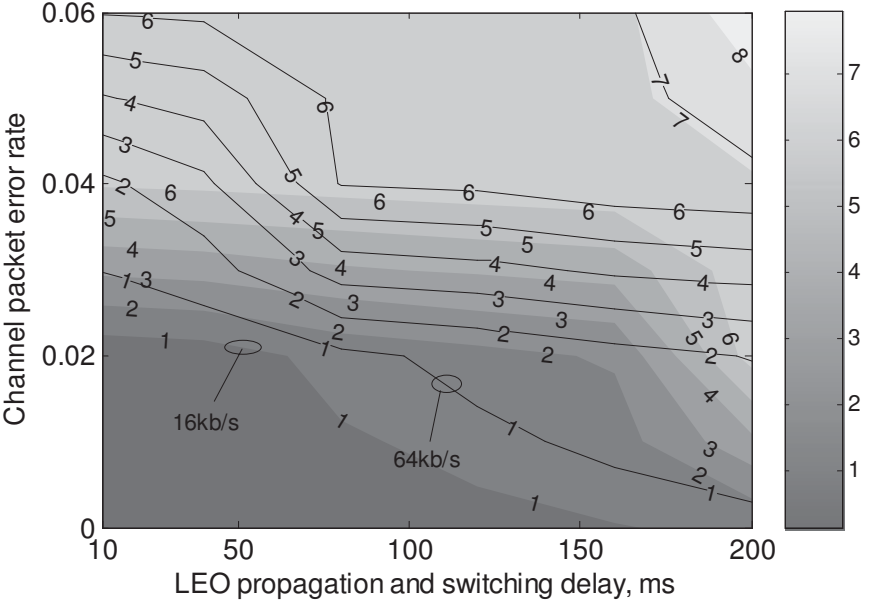

Fig. 6: Alarm message delivery duration $\left(\lambda=0.02 s^{-1}\right)$ : 16 $\mathrm{kb} / \mathrm{s}$ (filled contour plot), $64 \mathrm{~kb} / \mathrm{s}$ (solid lines)

minimal when the delay and error rate are high as the channel bandwidth is wasted with increased data transmission time.

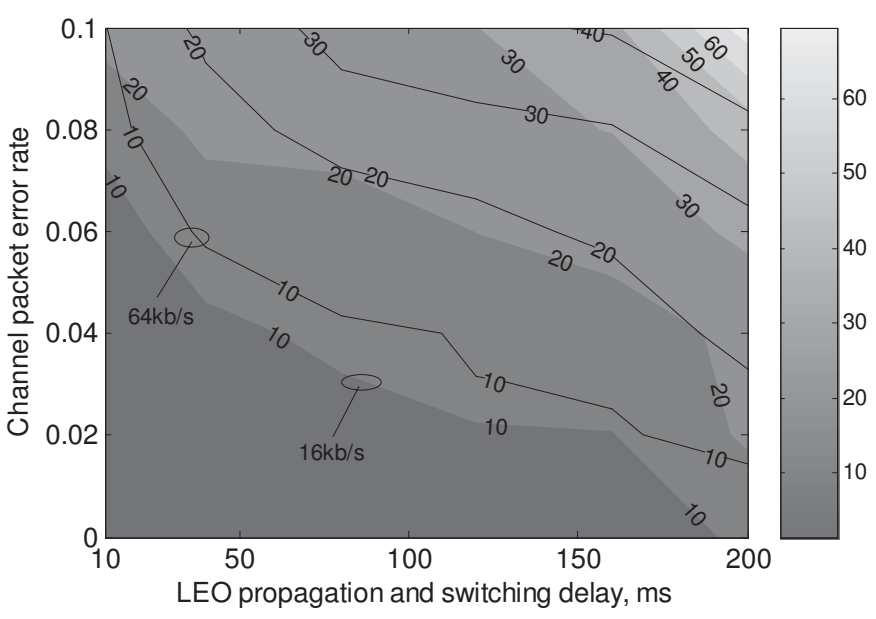

Fig. 7: Coordination data delivery duration $\left(\lambda=0.005 s^{-1}\right)$ : $16 \mathrm{~kb} / \mathrm{s}$ (filled contour plot), $64 \mathrm{~kb} / \mathrm{s}$ (solid lines)

Following the performance assessment with different channel capacity, we evaluate the LEO network when the power distribution network under anomalous and emergency conditions where the occurrence of field alarms and control actions is frequent. We consider that network monitoring is still periodic every $10 \mathrm{~s}$, but the alarms and control events are generated with the intensity of 0.2 and 0.05 (equivalent to the occurrence of every $5 \mathrm{~s}$ and $20 \mathrm{~s}$, respectively).

In our evaluation, no significant difference between the emergency condition and normal condition (as shown in Figure 4) for RTU data acquisition duration can be observed, so the result is not presented. Figure 8 illustrates the results of performance comparison between the emergency condition (solid lines) and the normal condition (filled contour plot) for control solution delivery with a forward and backward channel capacity of 2048 and $64 \mathrm{~kb} / \mathrm{s}$ respectively. It shows that it takes a longer time (a few extra seconds) to deliver the control 
commands under emergency conditions due to excessive traffic and the result tells us that the time requirement can be met for the $11 \mathrm{kV}$ network, even in the worst-case delay and loss scenario, under the anomalous network condition. In addition, reliable and low-latency terrestrial systems would be installed as backup to the satellite channels for certain critical $33 \mathrm{kV}$ sites, if feasible.

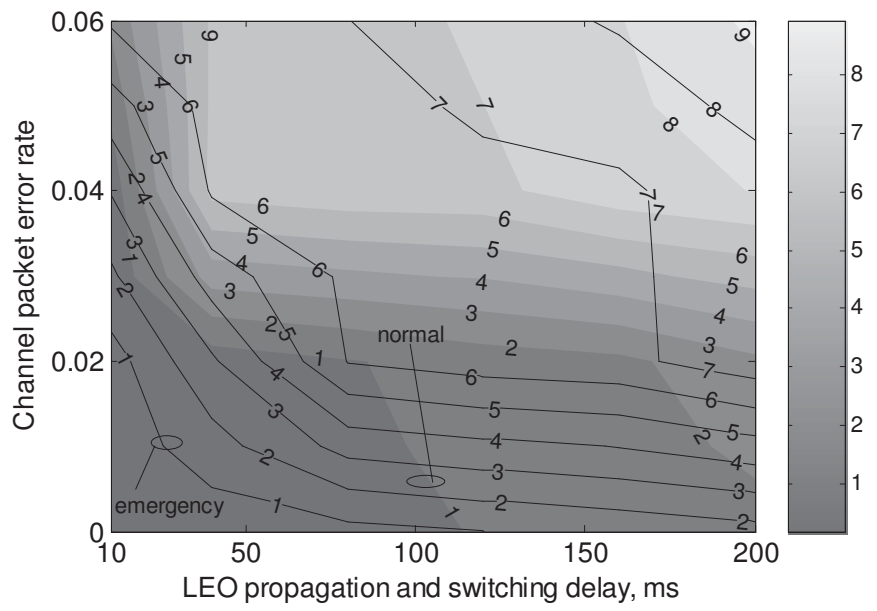

Fig. 8: Control command delivery duration: normal (filled contour plot) and emergency (solid lines) condition

Figure 9 illustrates the delivery performance of field alarms under the emergency condition when the arrival intensity is 0.2 compared to the normal condition $\left(\lambda=0.02 s^{-1}\right)$ with a channel capacity of $64 \mathrm{~kb} / \mathrm{s}$. It shows that if the packet error rate of the satellite channel is sufficient low, then the alarm packets can still be delivered within about $1 \mathrm{~s}$. Also, in the worst case scenario with the delay of $200 \mathrm{~ms}$ and error rate of 0.06 , the delivery time of the alarms is within $10 \mathrm{~s}$. The result indicates that the performance could meet the delivery time requirements under emergency operating conditions.

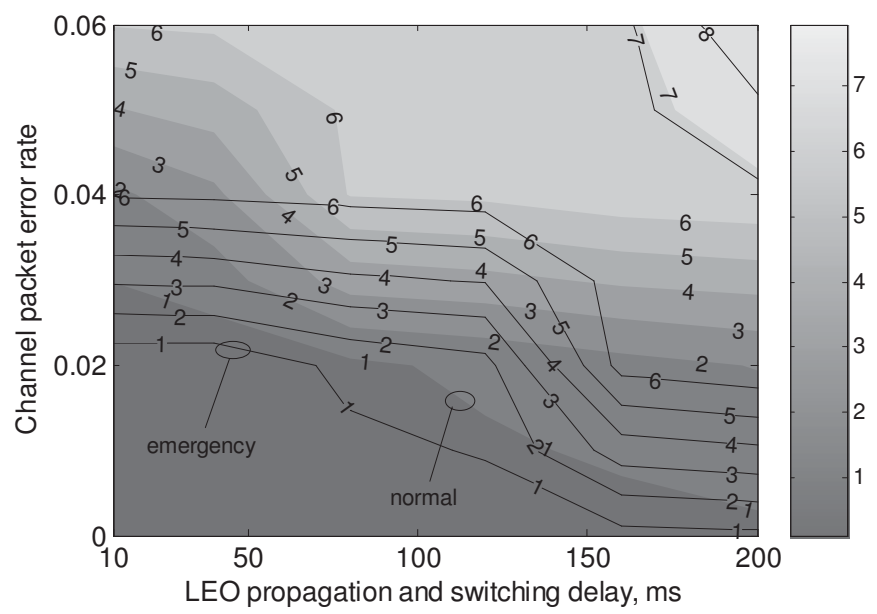

Fig. 9: Alarm delivery duration: normal (filled contour plot) and emergency (solid lines) condition

Finally, keeping the coordination event arrival intensity as $\lambda=0.005 s^{-1}$, we increase the number of exchanged ACL messages from 200 to 500 to represent a more sophisticated coordination process across multiple control regions. Figure 10 shows that in the worst case scenario, the data delivery duration for a coordination process is within $90 \mathrm{~s}$ (i.e. 1.5 minutes).

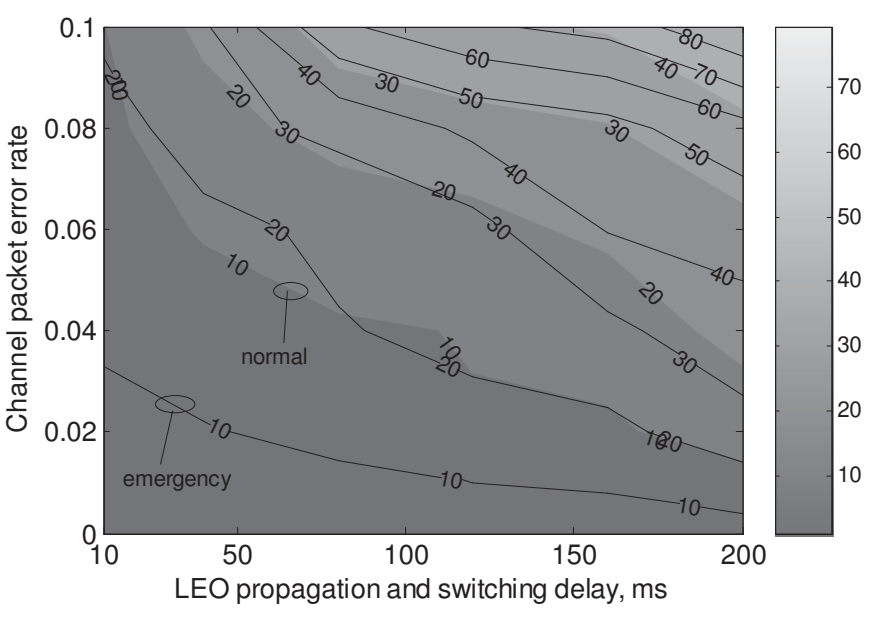

Fig. 10: Coordination data delivery duration: normal (filled contour plot) and emergency (solid lines) condition

\section{CONCLUSIONS AND Discussions}

This paper investigated the effectiveness of using standard IP based services over a "best effort" communication infrastructure using a LEO network as the key part for supporting a novel active network management solution of power distribution networks with DGs. The paper highlighted the major characteristics of LEO satellite networks and discussed their potential impact on delivering data traffic in the context of AURA-NMS. Rather than evaluating a specific LEO satellite constellation, we applied a generic approach to characterize the delay behavior of LEO constellations with an analytical model. Extensive simulation experiments for both normal and emergency network traffic scenarios against a wide range of delay and loss conditions are carried out and the results indicate that the data delivery timeliness of different traffic types could meet our expectations to support an AURANMS type of control solution in $33 \mathrm{kV}$ and $11 \mathrm{kV}$ distribution networks. It also should be noted that the adoption of advanced error control techniques or reliable backup communication channels in practical deployment could further promote the communication performance, and hence the functionality of an AURA-NMS type of approach.

Our key findings and remarks can be summarized as follows: (1) current SCADA systems in DNOs cannot meet the needs of active network management due to limited communication availability and bandwidth at medium voltage networks $(33 \mathrm{kV}$ and $11 \mathrm{kV})$, as highlighted in [5]. The adoption of a LEO network can well address this issue due to its large geographical coverage, including very remote sites, fast installation and flexible bandwidth allocation; (2) IP based services can operate well over the LEO network with the desired performance which offers an excellent opportunity for DNOs to migrate 
from their proprietary protocols and closed SCADA systems to a unified IP based future-proof open infrastructure to enable transparent communication across the overall distribution network, even across multiple utilities; (3) Properly interfacing with the legacy system is crucial to provide system backward compatibility and transparent communications service. Many techniques and solutions (e.g. [32], [33]) are available enabling satellite systems to flexibly and compatibly work in conjunction with various legacy wired/wireless terrestrial communication technologies with excellent interoperability and minimal reinforcement cost; (4) the main expenditure of adopting LEO technology in power utility includes the infrastructure cost (installation of ground transceivers) and monthly usage cost which are comparative low due to the low orbit and high frequency down-link which allows the use of small, low-cost, low-power terminals and antennas. Also the competition among satellite service providers keeps the monthly service price low. This is confirmed by [34] which presents a cost model of satellite system and compares the cost of satellite transceivers and service charges in using several different LEO constellations (including Iridium, Orbcomm, Globalstar and MSAT systems); and finally (5) LEO satellites are less expensive to build, but in general have a shorter designed average life span of 10-15 years. The life span of satellite constellations can be extended by periodic refueling and/or servicing of the satellites in the constellation and many strategies are available (e.g. [35]). In addition, power utilities could migrate to other satellite operators or service providers after the expiration of satellite constellation, which minimizes their risk of investments in equipments. In summary, we envisage that LEO satellite based systems can be served as the key component of the next-generation "utility Intranet" for managing the smart energy networks at the distribution level with the affordable cost.

As further empirical evidence from AURA-NMS type of solution becomes available, the outcome of additional assessment will be published. However, the results obtained so far are encouraging and worth further investigation. For example, the effect of adverse weather condition on the generic solution presented in this work could be a new area of further investigation.

\section{ACKNOWLEDGMENT}

This work was partially supported by the UK EPSRC (EP/E003583/1), ABB Limited, EDF Energy and SP Power Systems Limited under the AuRA-NMS research consortium, and the National Natural Science Foundation of China (grant No. 51107113).

\section{REFERENCES}

[1] G. P. Harrison and A. R. Wallace, "Maximising renewable energy integration within electrical networks," World Renewable Energy Congress, 22-27 May 2005, Aberdeen.

[2] IntelliGrid, "Smart power for the 21st century" [Online]. Available: http://www.intelligrid.epri.com/

[3] SmartGrids, "Vision and strategy for Europe's electricity networks of the future" [Online]. Available: http://www.smartgrids.eu/

[4] AuRA-NMS, "Autonomous regional active network management system" [Online]. Available: http://gow.epsrc.ac.uk/ViewGrant.aspx?GrantRef=EP/E003583/1
[5] Q. Yang, J. A. Barria, C. Hernandez Aramburo, "A communication system for regional control of power distribution networks," Proc. the 7th IEEE Int. Conf. on Industrial Informatics, pp.372-377, Cardiff, UK, June 2009.

[6] Q. Yang and J. Barria, "ICT system for managing medium voltage active distribution grids," Proc. the 35th Annual Conf. IEEE Industrial Electronics Society, pp. 3581-3586, Porto, Portugal, Nov. 3-5, 2009.

[7] Q. Yang, J. Barria, T. Green, "Communication infrastructures for distributed control of power distribution networks," IEEE Trans. Indu. Inform., vol. 7, no. 2., pp. 316-327, May 2011.

[8] K. P. Birman, et al., "Overcoming Communications Challenges in Software for Monitoring and Controlling Power Systems," Proc. the IEEE, vol. 93, no. 5, pp. 1028-1041, May 2005.

[9] K. Hopkinson, et al., "Quality of service considerations in utility communication networks," IEEE Trans. on Power Delivery, vol. 24, no. 3, pp. 1465-1474, July 2009.

[10] Y. S. N. Murty, "Hybrid communication networks for power utilities," Proc. The Power Quality, pp.239-242, Jun. 1998.

[11] K. E. Holbert, G. T. Heydt, and H. Ni, "Use of satellite technologies for power system measurements, command and control," Proc. the IEEE, vol. 93, no. 5, pp. 947 - 955, May 2005.

[12] D. J. Marihart, "Communication Technology Guidelines for EMS/SCADA systems," IEEE Trans. Power Deli., vol. 16, no. 2, pp. 181-188, April 2001.

[13] W. Usaha and J. A. Barria, "Reinforcement learning for resource allocation in LEO satellite networks," IEEE Trans. Syst., Man, Cybern., vol. 37 , no. 3 pp. $515-527$, Jun. 2007.

[14] Ekici E, Akyildiz IF, and Bender MD. "A distributed routing algorithm for datagram traffic in LEO satellite networks." IEEE/ACM Trans. Netw., vol. 9, no. 2, pp.137-147, April 2001.

[15] M. Marchese, M. Rossi, and G. Morabito, "PETRA: Performance enhancing transport architecture for satellite communications," IEEE J. Sel. Areas Commun., vol. 22, no. 2, pp. 320-332, Feb. 2004.

[16] Ozgur Ercetin, Srikanth Krishnamurthy, Son Dao, et al. "Provision of guaranteed service in broadband LEO satellite networks," Computer Networks, vol. 39, no. 1, pp. 61-77, 2002.

[17] T. Dhar and T. Tang, "Present and future communication technologies for distribution automation in rural Queensland, Australia," Proc. Int. Conf. Energy management and power Delivery, pp.650-655, Mar. 1998.

[18] A. Vaccaro and D. Villacci, "Performance analysis of low earth orbit satellites for power system communication," Electric Power Systems Research, vol. 73, no. 3, pp. 287-294, 2005.

[19] T. Xu, P. C. Taylor, et al. "Case-based reasoning for distributed voltage control," Proc. CIRED 2009, Prague, June 2009.

[20] M. J. Dolan, E. M. Davidson, et al. "Techniques for managing power flows in active distribution network within thermal constraints," Proc. CIRED 2009, Prague, June 2009.

[21] E. M. Davidson and S.D.J. McArthur, "Exploiting multi-agent system technology within an autonomous regional active network management system," Proc. Intelligent Systems Applications to Power Systems, pp. 1-6, Niigata, Nov. 2007.

[22] Lloyd's satellite constellations. Available: http://www.ee.surrey.ac.uk/Personal/L.Wood/constellations/

[23] F. Abrishamkar and Z. Siveski, "PCS global mobile satellites," IEEE Commun. Mag., vol. 34, no. 9, pp. 132-136, Sept. 1996.

[24] M. Allman, J. Griner, and A. Richard, "TCP behaviour in networks with dynamic propagation delay," Proc. IEEE GLOBECOM, pp. 1103 - 1108 , San Francisco, USA, Dec. 2000.

[25] H. Peyravi, "Medium Access Control protocols for space and satellite communications: a survey and assessment," IEEE Commun. Mag., vol. 37, no. 3, pp. 62-71, 1999

[26] D.C. Beste, "Design of satellite constellations for optimal continuous coverage," IEEE Trans. Aerosp. Electron. Syst., vol. 14, no. 3, pp. 466473, 1978.

[27] B. Gavish and J. Kalvenes, "The impact of satellite altitude on the performance of LEOS based communication systems," Wireless Networks vol. 4, no. 2, pp. 199-213, 1998.

[28] J. Zhu, S. Roy, and J. Kim, "Performance modelling of TCP enhancements in terrestrial-satellite hybrid networks," IEEE/ACM Trans. Netw., vol. 14, no.4, pp.753-766, August 2006.

[29] K. H. GjermundrØd, D. E. Bakken, C. H. Hauser, and A. Bose, "GridStat: A flexible QoS-managed data dissemination framework for the power grid," IEEE Trans. Power Del., vol. 24, no. 1, Jan. 2009.

[30] FIPA agent message transport protocol for HTTP specification, 2002 [Online] Available: http://www.fipa.org/specs/fipa00084/SC00084F.html

[31] Network simulator, [Online] Available: http:// www.isi.edu/nsnam/ns 
[32] B. Evans, "Integration of satellite and terrestrial systems in future multimedia communications," IEEE Wireless Communications, vol. 12, no. 5 , pp. 72-80, Oct. 2005.

[33] S. Kim, "Evaluation of cooperative techniques for hybrid/integrated satellite systems," Proc. IEEE International Conference on Communications, IEEE ICC, pp. 1-5, Kyoto, Japan, June 2011.

[34] B. Nickerson, A. Wu, "Cost model of satellite systems for real-time helicopter operations," Technical Report TR02-157, Faculty of Computer Science, University of New Brunswick, August 7, 2002, 26 pages.

[35] A. Dutta, P. Tsiotras, "A cooperative egalitarian peer-to-peer strategy for refueling satellites in circular constellations," AAS/AIAA Space Flight Mechanics Meeting, American Astronomical Society, Paper 09-109, Washington, D. C., Feb. 2009.

[36] IEEE, "Substation integrated protection, control and data acquisition communication requirements," IEEE Tech. Rep. P152522003, 2003, Draft. 